\title{
Efeito da velocidade do estímulo no desempenho de uma tarefa complexa de timing coincidente em crianças e adolescentes
}

\author{
Andrea Freudenheim \\ Jorge de Oliveira \\ Umberto Corrêa \\ Paula Oliveira \\ Luiz Dantas \\ Jane Silva \\ Cássia Moreira \\ Go Tani
}

\author{
Universidade de São Paulo \\ Escola de Educação Física e Esporte \\ Laboratório de Comportamento Motor \\ Brasil
}

\section{RESUMO}

O objetivo deste estudo foi verificar o efeito de diferentes velocidades de deslocamento do estímulo no desempenho de uma tarefa complexa de timing coincidente. No experimento 1, 42 crianças realizaram 25 tentativas na tarefa de tocar quatro alvos em integração a um estímulo visual, distribuídas em três grupos conforme as velocidades dos estímulos: lento $(1,11 \mathrm{~m} / \mathrm{s})$, moderado $(0,89 \mathrm{~m} / \mathrm{s})$ e rápido $(0,74 \mathrm{~m} / \mathrm{s})$. Não foi detectada diferença significativa $(\mathrm{p}<0,05)$ para a medida de erro absoluto. Para o erro variável, foi detectada diferença entre os grupos de velocidade rápida e moderada e entre os de velocidade moderada e lenta. No experimento 2, 49 adolescentes realizaram 25 tentativas em tarefa semelhante, distribuídos em três grupos em função da velocidade do estímulo $(2,02 \mathrm{~m} / \mathrm{s}, 1,70$ $\mathrm{m} / \mathrm{s}$ e $1,48 \mathrm{~m} / \mathrm{s})$. Não foram detectadas diferenças significativas entre os grupos. Conclui-se que numa tarefa complexa de timing coincidente, a velocidade do deslocamento do estímulo não afeta a precisão do desempenho de crianças e adolescentes, mas afeta a consistência do desempenho de crianças. Portanto, os resultados desse estudo não corroboram os da literatura baseados em estudos com tarefas simples de timing coincidente. No entanto, estudos adicionais necessitam ser realizados para explorar um espectro maior de velocidades de propagação do estímulo.

Palavras-chave: timing coincidente, desempenho, tarefas complexas.

\section{ABSTRACT \\ Effect of stimulus velocity on the performance of a complex coin- cident timing task with children and adolescents}

The purpose of the present study was to investigate the effect of different stimulus velocities on the performance of a complex coincident timing task. In Experiment 1, 42 children, distributed in groups according to stimulus velocity of $1,11,0,89$ or $0,74 \mathrm{~m} / \mathrm{s}$, underwent 25 trials. The set was to touch four keys in conjunction with a visual stimulus. No significant difference $(p<0,05)$ was detected in the absolute error measure. In relation to variable error significant differences were detected between the fast and moderate velocity groups, and between the slow and moderate velocity groups. In Experiment 2, 49 adolescents, distributed in groups according to stimulus velocity of 2,02, 1,70 or $1,48 \mathrm{~m} / \mathrm{s}$ had 25 trials in a similar task. No significant differ-ences were found. It can be concluded that stimulus velocity does not affect the precision of children and adolescents but affects consistency of children's performance in a complex coincident timing task. Therefore, the results of this study corroborate the results based on studies with simple coincidence timing tasks reported in literature. Nevertheless, additional studies should explore a broader stimulus velocity spectrum.

Key Words: coincident timing, performance, complex task. 


\section{INTRODUÇÃO}

A importância da capacidade de antecipação para a execução bem sucedida de ações motoras já foi bem documentada $(3,13,18)$. Sabe-se que a performance coordenada de um atleta interceptando uma bola, de um músico acompanhando uma orquestra, de um trabalhador industrial à frente de uma esteira ou de uma criança rebatendo uma bola, depende de iniciar a seqüência de movimentos no momento correto e de realizar as ações com precisão temporal. Mais especificamente, tarefas de timing coincidente requerem do executante uma previsão da posição futura de um objeto ou alvo móvel e da organização da resposta motora, de maneira a fazer o seu término coincidir com a chegada do estímulo externo ao local pré-determinado. Portanto, esse tipo de tarefa exige a capacidade de integrar a antecipação efetora e a receptora (17).

Vários estudos foram realizados com o intuito de conhecer o efeito de variáveis como idade, complexidade da resposta e velocidade do estímulo sobre o desempenho em tarefas dessa natureza. Os estudos que enfocaram a influência da idade demonstraram que a precisão em tarefas de timing coincidente aumenta com a idade $(1,4,6)$. Isso porque a antecipação receptora é um processo complexo que envolve simultaneamente componentes espaciais e temporais (12). Para realizar uma tarefa de timing coincidente com sucesso, o indivíduo deve ter a capacidade de antecipar o momento de ocorrência do evento (aspecto temporal) e a sua localização (aspecto espacial). Ele também precisa ser capaz de prever seus processos intrínsecos, como o tempo necessário para o processamento de informações e para a realização do movimento (1). Essas capacidades são adquiridas à medida que o mecanismo perceptivo, os processos de memória e o raciocínio desenvolvem-se. Aos nove anos de idade, a criança adquire condições de executar uma tarefa de timing coincidente, não com a mesma velocidade e precisão que um adulto, mas de forma bastante similar $(4,19)$. Somente aos 14 ou 15 anos de idade a curva de performance alcança uma assíntota, ou seja, a capacidade de antecipação torna-se equivalente à de adultos (4).

A maior parte dos trabalhos realizados para investigar o efeito de variáveis sobre o desempenho em tarefas de timing coincidente foi conduzida com tarefas sim- ples. A resposta motora mais freqüentemente usada é a de pressionar um botão no momento de coincidência com a chegada de um estímulo externo a um local específico (2). Movimentos de braço unidirecionais finalizando com o pressionar de um botão ou derrubada de um alvo também foram utilizados $(4,23,14)$. Em contraste, tarefas complexas do mundo real como o receber (16), o rebater (15) e o chutar (21) foram pouco utilizadas. No presente estudo, vale ressaltar que o nível de complexidade da tarefa está sendo diferenciado pelo número de graus de liberdade (articulações) que necessitam ser controlados para sua realização. Nesse sentido, a resposta de pressionar o botão é considerada simples, pois envolve poucos graus de liberdade a serem controlados.

Poucos estudos investigaram o efeito da complexidade da resposta no desempenho de tarefas de timing coincidente. Desses, alguns verificaram que o desempenho é mais preciso e consistente em tarefas simples do que em complexas $(6,11,22)$ e outros verificaram que não há correlação significante entre os resultados obtidos na tarefa simples de apertar o botão e na tarefa mais complexa de rebater (16) e de receber (5). No conjunto, esses resultados permitem concluir que não existe relação entre performances em tarefas simples e em tarefas complexas, uma vez que no caso de tarefas simples, a influência da antecipação efetora parece ser reduzida. Já na tarefa complexa, a demanda efetora aumenta e, em conseqüência, a influência da antecipação efetora também aumenta. Portanto, visto que a natureza das tarefas simples (baixa demanda efetora, alta demanda perceptiva) e complexas (com alta demanda efetora e perceptiva) é distinta (5), os resultados obtidos com tarefas simples não podem ser generalizados para as tarefas complexas e vice-versa.

Por sua vez, os estudos que enfocaram o efeito da velocidade do estímulo mostraram que a velocidade mais baixa de deslocamento induz à piora do desempenho $(13,20,23)$ e que há tendência de uma configuração em $U$ entre a velocidade do estímulo e a precisão da resposta. Esses estudos sugerem que é mais difícil responder a objetos rápidos do que lentos, mas que existe um momento em que o estímulo se move tão rapidamente que uma resposta precisa torna-se muito difícil $(13,20)$. Nesses estudos, realizados entre a década de 1970 e início da década de 
1990, foram utilizadas tarefas de timing coincidente simples, ou seja, com baixa demanda efetora.

Mais recentemente, Williams (21) realizou um estudo utilizando o chutar. Seu objetivo foi investigar o efeito da velocidade do estímulo luminoso (alta e baixa) e da extensão do movimento (um passo ou dois passos antes do chute) na precisão espacial e de timing coincidente no chute do futebol. Os participantes deveriam fazer a chegada da bola ao alvo coincidir com a chegada do estímulo luminoso ao final da seqüência de leds (diodos). Os resultados indicaram que o grupo que realizou a tarefa mais complexa (dois passos para o chute) na velocidade alta de propagação do estímulo foi menos preciso que os demais. Segundo o autor, esse resultado foi contrário ao esperado e ocorreu em razão de a velocidade do estímulo ter limitado a resposta, ou seja, ter significado uma restrição severa para a capacidade do sujeito de responder com precisão (21). $\mathrm{O}$ conjunto dos estudos remete à seguinte questão: em se tratando de uma tarefa complexa e não sendo a restrição temporal tão severa, qual seria o efeito da velocidade de propagação do estímulo no desempenho? Será que a velocidade mais baixa permaneceria a menos favorável?

Assim, considerando a importância de compreender as variáveis determinantes do desempenho em tarefas complexas de timing coincidente, o presente estudo buscou verificar o efeito de diferentes velocidades de deslocamento do estímulo no desempenho de uma tarefa complexa de timing coincidente em que vários alvos devem ser tocados, numa ordem pré-estabelecida, em integração a um estímulo visual. Considerando que a antecipação receptora é uma capacidade que se modifica ao longo do processo de desenvolvimento, foram realizados dois experimentos: um com a participação de crianças (Experimento 1) e outro com a participação de adolescentes (Experimento 2). Antes da realização de cada experimento foram efetuados estudos piloto para garantir que o nível de dificuldade da tarefa fosse adequado a cada amostra (10).

\section{EXPERIMENTO 1 \\ Método}

Participaram do estudo 42 crianças entre cinco e sete anos de idade de ambos os sexos, após apresentarem consentimento por escrito de seus responsáveis. Os dados foram coletados em duas escolas particulares brasileiras. A tarefa consistiu em tocar quatro alvos, numa ordem pré-estabelecida, em integração a um estímulo visual, portanto, envolvendo alta demanda efetora (tocar nos sensores na seqüência determinada) e receptora (fazer o toque no último sensor coincidir com o acendimento do último diodo).

$\mathrm{O}$ aparelho utilizado foi o timing coincidente em tarefas complexas (Patente $\mathrm{n}^{\circ}$ 0.403.1330-04), descrito a seguir e ilustrado na Figura 1. Ele é composto por uma canaleta com 100 diodos dispostos a uma distância de $1 \mathrm{~cm}$ uns dos outros, uma mesa de madeira sobre a qual ficam instalados os sensores, um computador e um software que controla a velocidade de propagação do estímulo.

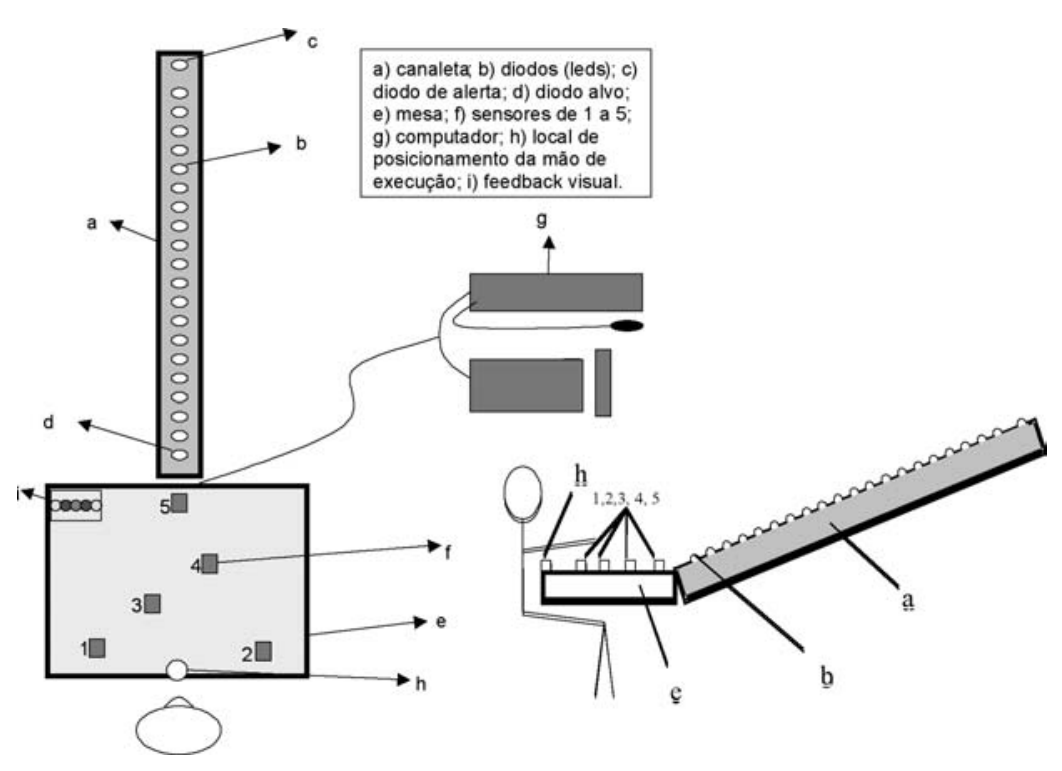

Figura 1 - Esquema ilustrativo do aparelho de timing coincidente em tarefas complexas. 
Os participantes foram distribuídos aleatoriamente em três grupos, conforme velocidade de propagação do estímulo: 20 crianças no grupo de velocidade rápida $(1,11 \mathrm{~m} / \mathrm{s}), 11$ crianças no de velocidade moderada $(0,89 \mathrm{~m} / \mathrm{s})$ e 11 crianças no de velocidade lenta $(0,74 \mathrm{~m} / \mathrm{s})$. Cada criança foi posicionada de frente para o aparelho, de pé, de forma que seu abdômen ficasse na altura da mesa, para que pudesse tocar todos os sensores. Após as explicações, o experimentador permitiu que a criança executasse a seqüência de toques até no máximo cinco vezes. Uma vez garantida a compreensão da tarefa, deu-se início à coleta. Cada criança realizou 25 tentativas numa mesma ordem de toques nos sensores (1-2-43). Após cada tentativa foi fornecida informação sobre o desempenho (conhecimento de resultado). O intervalo entre cada execução foi de aproximadamente seis segundos.

\section{Resultados}

Os erros dos executantes foram registrados em milisegundos. As medidas utilizadas foram: i) erro absoluto (EA), que corresponde à magnitude do erro, ou seja, à precisão do desempenho, e ii) o erro variável $(E V)$, que representa a consistência da resposta. Para a análise, como os dados não atenderam às condições de normalidade, a mediana calculada a partir das 10 tentativas finais foi utilizada como medida de tendência central, e para as comparações entre grupos foi utilizada a ANOVA de Kruskal Wallis e o teste de $U$ de Mann Whitney como post hoc.

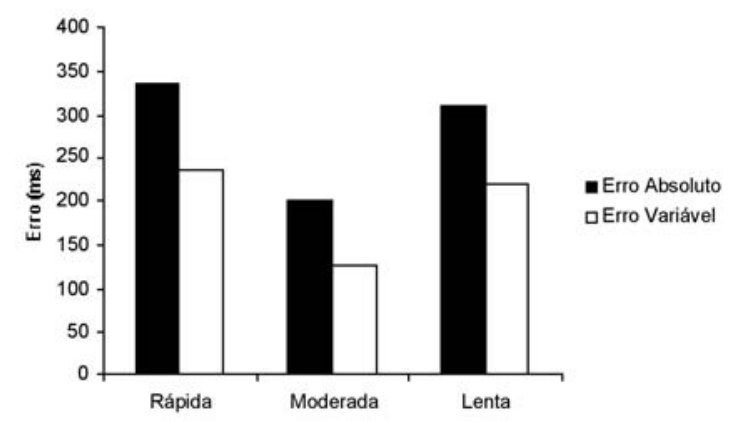

Figura 2 - Mediana dos erros absoluto e variável do desempenho na tarefa de timing coincidente complexo nas velocidades rápida, moderada e lenta em milissegundos ( $\mathrm{ms}$ ), do último bloco de 10 tentativas.
Como pode ser verificado na Figura 2, independentemente da velocidade de propagação do estímulo, as crianças foram pouco precisas (medianas acima de 200 ms) e muito variáveis (medianas acima de 100 $\mathrm{ms}$ ) em seu desempenho. Em ambas as medidas, pode-se observar a tendência a uma configuração em $U$, ou seja, de um melhor desempenho na velocidade moderada em relação às extremas. Na comparação entre os grupos, o teste de Kruskal Wallis não detectou diferença estatística significante para o EA [H $(2, \mathrm{~N}=$ $42)=4,47, p=0,10]$. Porém, na medida de erro variável, foram detectadas diferenças estatisticamente significativas entre os grupos $[\mathrm{H}(2, \mathrm{~N}=42)=7,72$; $\mathrm{p}=0,0210]$, e o teste de pos hoc localizou diferença entre os grupos de velocidade rápida e moderada e entre os grupos de velocidade moderada e lenta.

\section{Discussão}

Os resultados desse experimento reforçam a constatação de que crianças abaixo de nove anos de idade não têm ainda plenamente desenvolvida a sua capacidade de antecipação $(4,19)$. Além disso, a velocidade do deslocamento do estímulo não afetou a precisão do desempenho.

Uma possível explicação é a de que a dificuldade na realização da tarefa, mesmo tendo sido ajustada à faixa etária, superou o efeito da velocidade. Nem em termos de tendência se observa a superioridade da precisão na velocidade mais baixa verificada em vários estudos $(13,20,23)$, mas pode-se observar a tendência à configuração em $U(13,20)$. Assim, quando se trata de crianças efetuando uma tarefa complexa de timing coincidente, parece haver igualdade entre as velocidades com uma tendência de a velocidade moderada de propagação de estímulo ser a mais apropriada. No entanto, para verificar essas suposições, faz-se necessário estudos adicionais que explorem um espectro de velocidades maior do que o utilizado no presente estudo.

As crianças do grupo de velocidade moderada de propagação do estímulo foram mais consistentes em comparação com as crianças que praticaram nas velocidades rápida e lenta. Não houve diferença na consistência entre os grupos extremos. Nesse caso, a tendência à configuração do $U$ foi confirmada. Esse resultado indica que, nessa faixa etária, velocidades extremas (lentas e rápidas) dificultam ainda mais a realização da tarefa. 


\section{EXPERIMENTO 2 \\ Método}

Participaram deste experimento 49 adolescentes entre 15 e 16 anos de idade, alunos de uma escola particular brasileira. A tarefa, semelhante à utilizada no experimento 1 , consistiu em tocar cinco (em vez de quatro) sensores alvo, em uma ordem pré-estabelecida, fazendo o último toque coincidir com o acendimento do último diodo. As velocidades consideradas rápida, moderada e lenta foram, respectivamente, de $2,02 \mathrm{~m} / \mathrm{s}, 1,70 \mathrm{~m} / \mathrm{s}$ e $1,48 \mathrm{~m} / \mathrm{s}$. Essas modificações foram necessárias para ajustar o nível de complexidade da tarefa ao nível de desenvolvimento dos participantes, ou seja, para garantir a dificuldade funcional da tarefa (10). O aparelho foi o mesmo utilizado no experimento anterior. Os adolescentes foram distribuídos aleatoriamente em três grupos de velocidades distintas: 15 adolescentes no grupo de velocidade rápida $(2,02 \mathrm{~m} / \mathrm{s}) ; 15$ adolescentes no grupo de velocidade moderada $(1,70 \mathrm{~m} / \mathrm{s}) ; 19$ adolescentes no grupo de velocidade lenta $(1,48 \mathrm{~m} / \mathrm{s})$. O delineamento e os procedimentos foram os mesmos do experimento 1 .

\section{Resultados}

Como os dados das últimas 10 tentativas atenderam aos pressupostos de normalidade, a média foi utilizada como medida de tendência central e a comparação entre grupos foi realizada mediante análise de variância multivariada (MANOVA). Optou-se por um modelo multivariado porque as medidas de desempenho (erro absoluto e variável) foram consideradas em conjunto, por serem dependentes entre si (8).

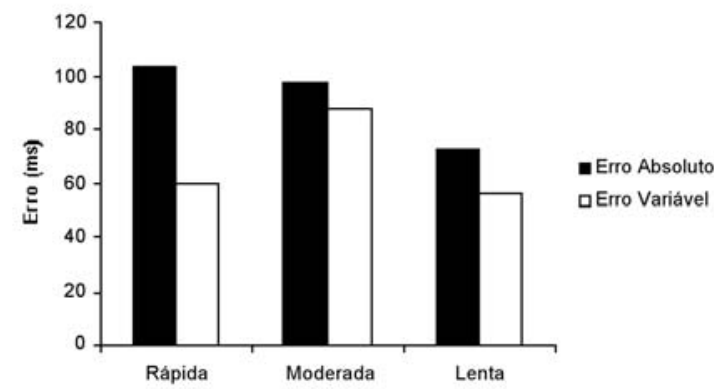

Figura 3 - Média dos erros absoluto e variável do desempenho na tarefa de timing coincidente complexo nas velocidades rápida, moderada e lenta em milissegundos (ms], do último bloco de 10 tentativas.
Como se pode observar na Figura 3, em relação à medida de precisão do desempenho (EA), há uma tendência de declínio conforme aumenta a velocidade de propagação do estímulo. Já a média do erro variável na velocidade moderada assemelha-se àquela das velocidades rápida e lenta. No entanto, não foram detectadas diferenças estatísticas significativas [Wilks' Lambda $=0,86, \mathrm{~F}(4 ; 72)=1,41, \mathrm{p}=0,24$ ].

\section{Discussão}

Os resultados desse experimento indicam que os adolescentes tiveram sucesso na realização da tarefa, pois foram precisos em seu desempenho. A média do erro absoluto foi próxima à de jovens adultos executando tarefa similar de alcançar e apertar um botão em coincidência com o acendimento do último diodo de uma seqüência, em uma velocidade próxima da efetuada (22). Portanto, conforme o esperado, a capacidade de antecipação dos adolescentes equivale à de jovens adultos (4).

A velocidade do deslocamento do estímulo não afetou a precisão do desempenho na execução da tarefa complexa de timing coincidente. Portanto, os adolescentes foram capazes de manter a precisão mesmo diante de modificações da tarefa. Os resultados também não indicaram tendência a um declínio no desempenho na velocidade mais baixa de deslocamento do estímulo ou mesmo nas velocidades extremas (configuração do $U$ ), como apontado nos estudos que utilizaram tarefas simples de timing coincidente $(13,20,23)$. Ao contrário, de acordo com os resultados, houve uma tendência linear de declínio da precisão do desempenho em virtude do aumento da velocidade de propagação do estímulo. Portanto, nesse aspecto, os achados apontaram na direção do resultado considerado anômalo por Williams (21). Na medida de EV também não foi detectada diferença estatística, pois nas três velocidades de propagação do estímulo a variabilidade das respostas foi equivalente.

Em conjunto, os resultados do experimento 2 mostraram que para adolescentes executando uma tarefa complexa de timing coincidente não há diferença no desempenho (EA e EV) entre grupos de velocidades distintas. Esses resultados não corroboraram os dos estudos realizados com tarefas simples de timing coincidente. 
Uma possível explicação remete à capacidade de adaptação do ser humano ante modificações do ambiente. O comportamento motor habilidoso, além de consistente, é flexível por natureza.

Principalmente em habilidades abertas, como as de timing coincidente, os estímulos ambientais relevantes mudam exigindo modificações no nível da tomada de decisão e da programação motora. Nessas tarefas, o processo de aquisição compreende desenvolver um vasto repertório de respostas para poder diversificar a maneira de alcançar a meta desejada (9). Assim, uma vez adquirida a habilidade, o executante possui a certeza de que o resultado será alcançado repetidas vezes, mesmo que o ambiente esteja em constante mudança. Essa certeza não implica rigidez do comportamento, mas um grau de variação comportamental necessário para manter a estabilidade do desempenho (7). Dessa forma, é possível que a diferença entre as velocidades utilizadas nesse estudo não tenha ido além da capacidade de adaptação dos participantes às mudanças ambientais. Essa suposição merece ser mais bem investigada em trabalhos futuros.

Possivelmente, a relação entre velocidade e desempenho não é direta como relatada na literatura.

Portanto, para que se possa conhecer melhor o efeito de diferentes velocidades de propagação do estímulo no desempenho motor de adolescentes é imprescindível que estudos adicionais explorem um espectro maior de velocidades de propagação do estímulo.

\section{CONCLUSÃO}

Os resultados do Experimento 1 permitem concluir que:

a) as crianças de cinco a sete anos de idade têm dificuldade de realizar tarefas complexas de timing coincidente;

b) a velocidade do deslocamento do estímulo não afetou a precisão do desempenho, indicando que a dificuldade na realização da tarefa supera o efeito da velocidade na precisão do desempenho; e,

c) a velocidade afetou a consistência da resposta de acordo com a configuração do $U$, indicando que, nessa faixa etária, velocidades extremas (lentas e rápidas) dificultam ainda mais o desempenho quando se trata de tarefas complexas de timing coincidente.
Os resultados do Experimento 2, por sua vez, permitem concluir que:

a) a capacidade de antecipação dos adolescentes equivale à de jovens adultos;

b) a velocidade do deslocamento do estímulo não afeta a precisão nem a consistência do desempenho na tarefa complexa de timing coincidente; e,

c) as velocidades utilizadas nesse estudo não foram além da capacidade de adaptação dos adolescentes às mudanças ambientais e que esse aspecto merece ser investigado em trabalhos futuros.

No seu conjunto, os resultados desse estudo, envolvendo crianças e adolescentes como sujeitos, não corroboram os achados da literatura, amplamente baseados em tarefas simples de timing coincidente. Por esse motivo, é importante a realização de estudos adicionais para explorar um espectro maior de velocidades de propagação do estímulo.

\section{Agradecimento}

Pesquisa fomentada pelo CNPq (015886/2002-6).

\section{CORRESPONDÊNCIA}

Andrea Michele Freudenheim

Universidade de São Paulo

Escola de Educação Física e Esporte

Laboratório de Comportamento Motor

Av. Prof. Mello Moraes, 65

CEP 05508-900 São Paulo - SP- BRASIL

amfreud@usp.br 


\section{REFERÊNCIAS BIBLIOGRÁFICAS}

1. Bard C, Fleury M, Gagnon, M (1990). Coincidence-anticipation timing: an age related perspective. In: C Bard, $M$ Fleury, L Hay (ed.) Development of eye-handcoordination across life span. Columbia: University of South Carolina, 283-305.

2. Brady F (1996). Anticipation of coincidence, gender, and sports classification. Perceptual and Motor Skills, 82(3): 227239.

3. Conrad R (1955). Timing. Occupational Psychology, 29: 173181.

4. Dorfman PW (1977). Timing and anticipation: a developmental perspective. Journal of Motor Behavior, 9: 67-79.

5. Ferraz, OL (1993). Desenvolvimento de timing antecipatório em crianças. Tese Mestrado, Escola de Educação Física da Universidade de São Paulo, São Paulo.

6. Fleury M, Bard C (1985). Age, stimulus velocity and task complexity as determiners of coincident timing behavior. Journal of Human Movement Studies, 11: 305-317.

7. Freudenheim AM (2005). Estabilidade e variabilidade na aquisição de habilidades motoras. In: G. Tani (ed.) Comportamento motor: aprendizagem e desenvolvimento. Rio de Janeiro: Guanabara Koogan, 117-128.

8. Green SB, Salkind NJ, Akey TM (2000). Using SPSS for Windows: analyzing and understanding data (2ed). New Jersey: Prentice Hall, 198-207.

9. Gentile AM (1972). A working model of skill acquisition with application to teaching. Quest, 17: 3-23.

10. Guadagnoli MA, Lee TD (2004). Challenge point: a framework for conceptualizing the effects of various practice conditions in motor learning. Journal of Motor Behavior, 36(2): 212-224.

11. Haywood KM (1977). Eye movements during coincidenceanticipation performance. Journal of Motor Behavior, 9: 313318.

12. Kerr R, Blais C (1991). Nonballistic coincident timing. Perceptual and Motor Skills, 71(1), 161-162.

13. Magill RA (1989). Motor learning: concepts and applications (3a. ed). Dubuque, Iowa: Wm C.Brown, 159-169.

14. Magill RA, Chamberlin CJ, Hall, KG (1991). Verbal knowledge of results as a redundant information for learning an anticipation timing skill. Human Movement Science, 10: 485-502.

15. Meeuwsen HJ, Goode SL, Goggin NL (1995). Coincidenceanticipation timing. Women in Sport and Physical Activity, 4: 59-75.

16. Petrakis, E (1985). Sex differences and specificity of anticipation of coincidence. Perceptual and Motor Skills, 61(3): 1135-1138.

17. Poulton EC (1957). On prediction in skilled movements. Psychological Bulletin, 54: 467-68.

18. Schmidt RA (1968). Anticipation and timing in human motor performance. Psychological Bulletin, 70: 63-46.

19. Stadilus RE (1985). Coincidence-anticipation behavior of children. In: JE Clark, JH Humphrey (eds.) Motor development: current selected research. Princeton: Princeton Book, 1-17.

20. Teixeira LA, Santos VA, Andreysuk R (1992). Tarefas que envolvem timing antecipatório: seriam as velocidades mais baixas as mais fáceis para sincronizar? Revista Paulista de Educação Física, 6(2): 21-28.

21. Williams LRT (2000). Coincidence timing if a soccer pass: effects of stimulus velocity and movement distance. Perceptual and Motor Skills, 91: 39-52.
22. Williams LRT, Jasiewicz J, Simmons RW (2001) Coincidence timing of finger, arm, and whole body movements. Perceptual and Motor Skills, 92: 535-547.

23. Wrisberg CA, Hardy CJ, Beitel PA (1982). Stimulus velocity and movement distance as determiners of movement velocity and coincident timing accuracy. Human Factors, 24(5): 599-608. 